Copyright (C 2008 IEEE. Reprinted from IEEE Photonics Technology Letters, 2007; 19 (15):1112-1114

This material is posted here with permission of the IEEE. Such permission of the IEEE does not in any way imply IEEE endorsement of any of the University of Adelaide's products or services. Internal or personal use of this material is permitted. However, permission to reprint/republish this material for advertising or promotional purposes or for creating new collective works for resale or redistribution must be obtained from the IEEE by writing to pubs-permissions@ieee.org.

By choosing to view this document, you agree to all provisions of the copyright laws protecting it. 


\title{
Low Walk-Off Kerr-Shutter Using a Dispersion-Shifted Lead Silicate Holey Fiber
}

\author{
S. Asimakis, G. Meloni, J. Y. Y. Leong, F. Poletti, R. C. Moore, K. E. Frampton, X. Feng, W. H. Loh,
} A. Bogoni, L. Potì, D. J. Richardson, and P. Petropoulos

\begin{abstract}
We present, for the first time to our knowledge, the use of a dispersion-shifted soft-glass holey fiber (HF) in a Kerr-shutter configuration. Wavelength conversion of $10-\mathrm{Gb} / \mathrm{s}$ data pulses is achieved in the $C$-band using just $2.1 \mathrm{~m}$ of a lead-silicate $\mathrm{HF}$ with a nonlinear parameter $\gamma$ of $164 \mathrm{~W}^{-1} \cdot \mathbf{k m}^{-1}$. The low dispersion and the short length of the fiber enable short switching windows to be realized.
\end{abstract}

Index Terms-Nonlinear optics, optical fiber communication, optical Kerr effect, photonic crystal fibers.

\section{INTRODUCTION}

C ROSS-PHASE modulation (XPM)-induced nonlinear polarization rotation has long been exploited for the implementation of ultrafast all-optical switches through a commonpath configuration widely known as the Kerr-shutter (KS) [1]. The optical fiber is considered as a particularly attractive nonlinear medium for the utilization of the Kerr effect and hence for the realization of a KS, since it can combine a high-power density with long interaction lengths. Thus, such applications as demultiplexing [2], [3], switching [4], and wavelength conversion (WC) [5], [6] based on KSs have already been demonstrated. An ideal fiber for KS applications would simultaneously exhibit good polarization stability, a low dispersion and a low walk-off between the pump and the probe pulses [1]. Besides, it is obvious that shorter fiber lengths are less sensitive to environmental variations, which can cause timing fluctuations and polarization instabilities, and they also lead to decreased net dispersion and walk-off. In order to achieve substantial polarization rotation in a short fiber length, a high effective nonlinear coefficient is essential. Indeed, some of the most promising demonstrations of KS switching have relied on the use of soft-glass highly nonlinear fibers (SG-HNLFs) [2], [4], [5], [7]. The ultrahigh nonlinearity of such fibers has enabled the realization of compact and stable devices employing just a few meters of fiber. Nevertheless, the conventional step-index design does not

Manuscript received December 11, 2006; revised April 22, 2007. This work was supported in part by E.U. NoE e-Photon/One. The work of S. Asimakis was supported by the Greek State Scholarships Foundation (I.K.Y.).

S. Asimakis, J. Y. Y. Leong, F. Poletti, K. E. Frampton, X. Feng, W. H. Loh, D. J. Richardson, and P. Petropoulos are with the Optoelectronics Research Center, University of Southampton, Southampton, SO17 1BJ, U.K. (e-mail: sya@orc.soton.ac.uk).

R. C. Moore is with the Centre of Expertise in Photonics, University of Adelaide, Adelaide 5005, Australia (e-mail: pp@orc.soton.ac.uk).

G. Meloni, A. Bogoni, and L. Potì are with the Integrated Research Center for Photonic Networks and Technologies, Scuola Superiore Sant'Anna, 56124 Pisa, Italy (e-mail: g.meloni@ sssup.it).

Digital Object Identifier 10.1109/LPT.2007.900309

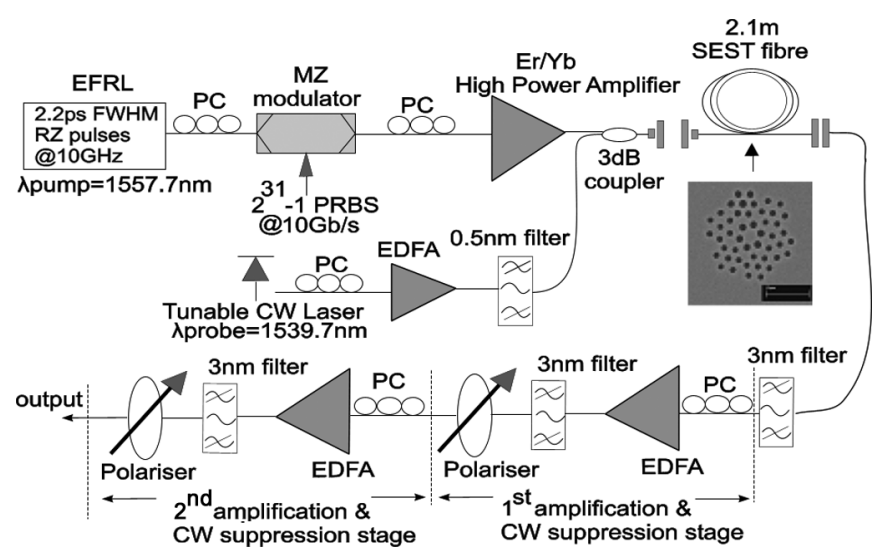

Fig. 1. Experimental setup. PC: polarization controller.

allow for flexible dispersion adjustment, thus these fibers usually suffer from a high normal dispersion and a steep dispersion slope in the $1.55-\mu \mathrm{m}$ window, which is a characteristic of glasses with a high nonlinear refractive index. As a result, pulse broadening and a large pump-probe walk-off hinder the implementation of short switching windows.

In our approach, we also use a nonsilica highly nonlinear glass to ensure a high nonlinear coefficient, but fabricate our fibers using holey fiber (HF) technology, which allows for flexible control of the dispersion properties. In this letter, we report the first demonstration of a KS using a lead-silicate HF (SF57-HF) that exhibits a low dispersion in the $C$-band and a nonlinearity of $164 \mathrm{~W}^{-1} \cdot \mathrm{km}^{-1}$. Using just $2.1 \mathrm{~m}$ of this fiber, WC of 2.2-ps pulses at $10 \mathrm{~Gb} / \mathrm{s}$ is realized. The low fiber dispersion leads to negligible walk-off between the original and the switched signals, and ensures that the pulsewidth of the converted output is the same as that of the original data pulses.

\section{EXPERIMENTAL SETUP}

The experimental setup is shown in Fig. 1. The transmitter at the pump port was based on a 10-GHz actively mode-locked erbium fiber ring laser which generated $\sim 2$.2-ps full-width at half-maximum (FWHM) soliton pulses at a central wavelength of $1557.7 \mathrm{~nm}$. The pulses were modulated with a $2^{31}-1$ pseudorandom bit sequence using a lithium-niobate Mach-Zehnder modulator and were amplified by an $\mathrm{Er}-\mathrm{Yb}$ amplifier. In the probe port, a continuous-wave $(\mathrm{CW})$ laser operating at $1539.7 \mathrm{~nm}$ was used as the source. The probe beam was amplified by a separate erbium-doped fiber amplifier (EDFA). A bandpass filter (BPF) was employed to remove the out-of-band 
amplified-spontaneous emission (ASE) noise resulting from the amplification process. The probe was combined with the pump signal through a 3-dB fiber coupler. The combined beam was free-space launched into the 2.1-m-long SF57-HF with a coupling efficiency of $\sim 27 \%$. The average powers of the pump and probe signals at the input of the fiber were then 17.6 and $13.0 \mathrm{dBm}$, respectively.

The fiber employed in the experiment was fabricated from the commercial lead-silicate Schott glass SF57 using the structured-element stacking technique (SEST), which allows for the realization of designs with novel dispersion properties. The design and fabrication of this fiber was reported in [8]. The core diameter of the fiber, as obtained from scanning electron microscope images, was $\sim 4.3 \mu \mathrm{m}$. The propagation loss and the nonlinear coefficient $\gamma$ of the fiber were determined experimentally to be $3.2 \mathrm{~dB} / \mathrm{m}$ and $164 \mathrm{~W}^{-1} \cdot \mathrm{km}^{-1}$, respectively [9]. The ability of the fiber to maintain linear polarization was also tested. By launching light on one of the principal polarization axes of the SF57-HF, we measured an extinction ratio of $\sim 22 \mathrm{~dB}$ between the signals received on the two orthogonal polarization axes at the output of the fiber. The beat length of the fiber was experimentally determined to be $\sim 0.6 \mathrm{~cm}$, yielding a differential group delay (DGD) of $0.86 \mathrm{ps} / \mathrm{m}$. From separate experiments on four-wave mixing in this fiber, we estimated a zero-dispersion wavelength of $1582 \mathrm{~nm}$ and a dispersion slope of $0.2 \mathrm{ps} / \mathrm{nm}^{2} \cdot \mathrm{km}$ within the $C$-band [9]. This combination of a low dispersion (with a relatively low dispersion slope), high effective nonlinearity, and good polarization-maintaining properties, makes this SF57-HF ideal for KS applications.

The arrangement of our KS followed the standard configuration. Two polarization controllers at the pump and probe ports were used for adjustment of the state of polarization of the two beams. The polarization of the pump beam was aligned to one of the principal polarization axes of the fiber, while the probe beam was linearly polarized at the SF57-HF input at $45^{\circ}$ relative to the pump. The presence of the pump pulses induced an intensity-dependent rotation of the state of polarization of the probe beam. At the output of the SF57-HF, the pump was filtered from the probe using a 3-nm BPF and subsequently, the probe signal passed through two successive amplification and polarization-filtering stages, each consisting of a polarization controller, an EDFA, a BPF, and a polarizer. The polarizers ensured that the Kerr-induced polarization rotation of the probe was transformed into intensity modulation. This was achieved by adjusting the polarization controllers at the input, so that transmission of the probe through the polarizers was minimized in the absence of the pump. The output power of each EDFA was $\sim 9.0 \mathrm{dBm}$. It should be noted that the two successive stages of polarization-filtering the signal were necessary, since the polarizers employed in our setup had a limited extinction ratio of 24 and $18 \mathrm{~dB}$, respectively. The BPFs used in these stages ensured that both the ASE of the two EDFAs and the pump signal were further suppressed. Thus, the pump was effectively suppressed at the output of our system.

\section{EXPERIMENTAL RESULTS AND DISCUSSION}

The received spectrum at the output of the SF57-HF is shown in Fig. 2(a). Clear sidebands arising from XPM are observed at the probe wavelength. For comparison, the probe spectrum at the input is also shown. In Fig. 2(b), the received spectra after
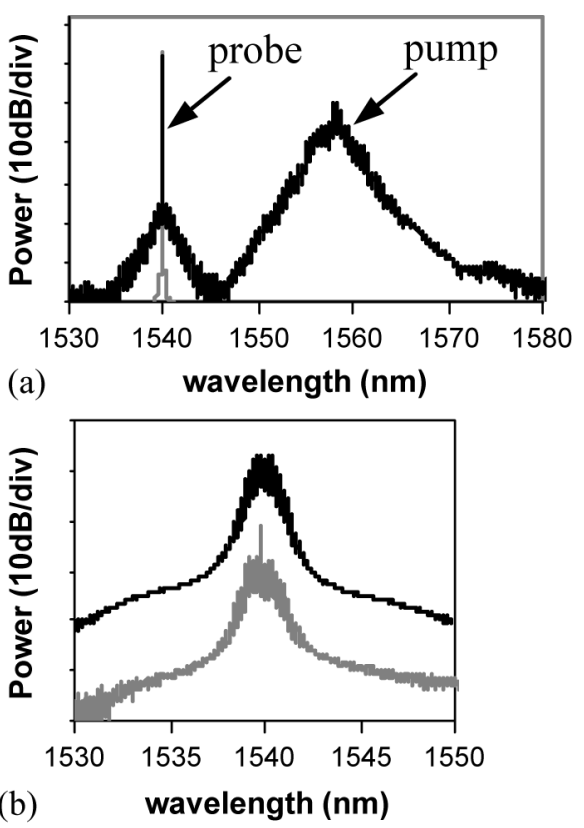

Fig. 2. (a) Spectrum after the SF57-HF when both the pump and the probe are launched (black trace) and input probe spectrum (gray trace); (b) received spectra after the first polarizer (gray trace) and at the output of the system (black trace).

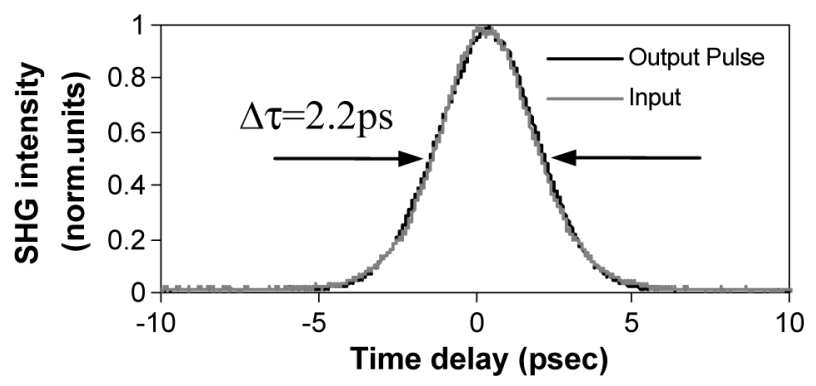

Fig. 3. SHG autocorrelation traces of the input and output pulses of the KS.

each of the two polarizers are shown. It is evident that effective suppression of the $\mathrm{CW}$ component of the probe was achieved after the combination of the two polarizers.

We next measured the pulsewidth at the output of the KS through second-harmonic generation (SHG) autocorrelation measurements, and compared it against the width of the input laser pulses (Fig. 3). No pulse broadening was observed, and a pulse FWHM of 2.2 ps was measured at the output. Numerical simulations performed on the KS system, for which the dispersive, nonlinear, and DGD properties of the SF57-HF were taken into account, confirmed this short switching window. This can be understood by considering the combination of the low dispersion of the fiber, which did not lead to substantial broadening of the gating pulse, and its low dispersion slope, which yields a walk-off length of $\sim 12$ m, i.e., much larger than the physical length of the SF57-HF. (Note that, even if the wavelength separation between the pump and probe had been $30 \mathrm{~nm}$, the walk-off length would be $6 \mathrm{~m}$ ). The significance of these figures can be appreciated when compared to the values exhibited by other SG-HNLFs. For example, the walk-off length of a step-index bismuth-oxide fiber, similar to the one used in [5], [10] would be just $\sim 30 \mathrm{~cm}$ for the same conditions 

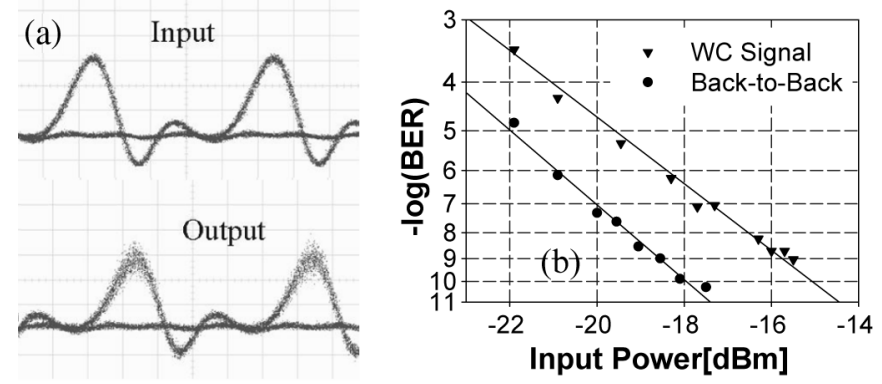

Fig. 4. (a) Eye diagrams of the input and output pulses; (b) corresponding BER curves.

as those considered in our experiment, and the pulsewidth at the output would inevitably be broadened in KS devices of a similar length to those considered here. (This pulse broadening has actually been experimentally observed in [5].) A comparison with conventional germanosilicate HNLF technology is not as straightforward. The high degree of control of dispersion and dispersion slope offered by this mature technology means that walk-off issues and the effects of dispersion can largely be eliminated. However, due to the relatively low nonlinear coefficient of conventional HNLFs, far longer fiber lengths are in general required, with implications on the effects of environmental stability and latency of the switching system.

The performance of the SF-57-HF wavelength converter was assessed both in terms of eye diagrams and bit-error-rate (BER) tests. In Fig. 4(a), the eye diagrams obtained at the input of the system are compared to the received eye diagrams at the output of the KS. A clear converted eye diagram is observed, albeit with some intensity noise, which we attribute to the accumulation of ASE through the two amplification stages at the output of the system. The effects of ASE are also present in the BER tests shown in Fig. 4(b). The WC process has introduced a power penalty of $\sim 3 \mathrm{~dB}$ at a BER of $10^{-9}$. However, some power leakage from the original $\mathrm{CW}$ probe could also be associated with the observed power penalty. Note that the normal dispersion of the SF57-HF at the operating wavelengths discourages the development of any modulation instability effects that might affect the inherent noise performance of the KS.

The SEST fabrication technique allows the implementation of dispersion-optimized soft-glass HF designs that exhibit even higher values of $\gamma$ than the fiber used in these experiments. Furthermore, even lower values of dispersion and dispersion slope within the $C$-band are possible [9], thus enabling a further reduction in the achievable length of the switching windows. In addition, previous fabrication attempts [11] have revealed that losses as low as $2 \mathrm{~dB} / \mathrm{m}$ should readily be achievable in this glass, further reducing the requirements in terms of device length/switching power. Finally, the optical signal-to-noise ratio in our experiments has been severely compromised by the nonoptimum light coupling into and out of the SF57-HF. It is expected that more thorough elaboration on the coupling between the SF57-HF and the SMF patch-cords will greatly improve the noise performance in future KS configurations.

\section{CONCLUSION}

A KS operating at $10 \mathrm{~Gb} / \mathrm{s}$ in the $1.55-\mu \mathrm{m}$ window and utilizing just $2.1 \mathrm{~m}$ of a dispersion-shifted SF57-HF was demonstrated. The combination of a short fiber length and a low dispersion results in a walk-off-insensitive performance, allowing for short switching windows to be realized. The fabrication of our fiber benefited from the SEST technique, which allows sophisticated microstructure designs to be implemented using simple extrusion processes for the fabrication of the preform. We believe that this experiment highlights the potential of soft-glass $\mathrm{HF}$ technology for the realization of compact and ultrafast nonlinear devices.

\section{ACKNOWLEDGMENT}

The authors wish to acknowledge the contribution of Prof. T. M. Monro and Dr. H. Ebendorff-Heidepriem to the development of the SEST fabrication technique.

\section{REFERENCES}

[1] J. M. Dziedzic, R. H. Stolen, and A. Ashkin, "Optical Kerr effect in long fibers," Appl. Opt., vol. 20, pp. 1403-1406, 1981.

[2] G. Meloni, M. Scaffardi, P. Ghelfi, A. Bogoni, L. Potì, and N. Calabretta, "Ultrafast all-optical ADD-DROP multiplexer based on 1-m-long bismuth oxide-based highly nonlinear fiber," IEEE Photon. Technol. Lett., vol. 17, no. 12, pp. 2661-2663, Dec. 2005.

[3] S. Watanabe, R. Okabe, F. Futami, R. Haindberger, C. SchmidtLanghorst, C. Schubert, and H. G. Weber, "Novel fiber Kerr-switch with parametric gain: Demonstration of optical demultiplexing and sampling up to $640 \mathrm{~Gb} / \mathrm{s}$," presented at the Eur. Conf. Optical Communication, Stockholm, Sweden, Sep. 5-9, 2004, Paper Th4.1.6.

[4] M. Asobe, T. Kanamori, and K. Kubodera, "Applications of highly nonlinear chalcogenide glass fibers in ultrafast all-optical switches," IEEE J. Quantum Electron., vol. 27, no. 8, pp. 2325-2332, Aug. 1993.

[5] J. H. Lee, K. Kikuchi, T. Nagashima, T. Hasegawa, S. Ohara, and N. Sugimoto, "All-fiber 80-Gbit/s wavelength converter using 1-m-long bismuth oxide-based nonlinear optical fiber with a nonlinearity $\gamma$ of $1100 \mathrm{~W}^{-1} \cdot \mathrm{km}^{-1}$," Opt. Express, vol. 13, pp. 3144-3149, 2005.

[6] G.-W. Lu, L.-K. Chen, C.-K. Chan, and C. Lin, "All-optical tunable wavelength conversion based on cross-polarisation modulation in nonlinear photonic crystal fibre," Electron. Lett., vol. 41, pp. 203-205, 2005.

[7] J. H. Lee, T. Nagashima, T. Hasegawa, S. Ohara, N. Sugimoto, and K. Kikuchi, "Wide-band tunable wavelength conversion of $10-\mathrm{Gb} / \mathrm{s}$ nonreturn-to-zero signal using cross-phase-modulation-induced polarization rotation in 1-m bismuth oxide-based nonlinear optical fiber," IEEE Photon. Technol. Lett., vol. 18, no. 1, pp. 298-300, Jan. 1, 2006.

[8] J. Y. Y. Leong, S. Asimakis, F. Poletti, P. Petropoulos, X. Feng, R. Moore, K. Frampton, T. M. Monro, H. Ebendorff-Heidepriem, W. Loh, and D. J. Richardson, "Towards zero dispersion highly nonlinear lead silicate glass holey fibres at $1550 \mathrm{~nm}$ by structured-element-stacking," presented at the Eur. Conf. Optical Communication, Glasgow, U.K., Sep. 25-29, 2005, Paper Th4.4.5, (postdeadline).

[9] S. Asimakis, P. Petropoulos, F. Poletti, J. Y. Y. Leong, R. C. Moore, K. E. Frampton, X. Feng, W. H. Loh, and D. J. Richardson, "Towards efficient and broadband four-wave-mixing using short-length dispersion tailored lead silicate holey fibres," Opt. Express, vol. 15, pp. 596-601, 2007.

[10] F. Parmigiani, S. Asimakis, N. Sugimoto, F. Koizumi, P. Petropoulos, and D. J. Richardson, " $2 \mathrm{R}$ regenerator based on a 2-m-long highly nonlinear bismuth oxide fiber," Opt. Express, vol. 14, pp. 5038-5044, 2006.

[11] J. Y. Y. Leong, P. Petropoulos, J. H. V. Price, H. Ebendorff-Heidepriem, S. Asimakis, R. C. Moore, K. E. Frampton, V. Finazzi, X. Feng, T. M. Monro, and D. J. Richardson, "High-nonlinearity dispersionshifted lead-silicate holey fibers for efficient $1-\mu \mathrm{m}$ pumped supercontinuum generation," J. Lightw. Technol., vol. 24, no. 1, pp. 183-190, Jan. 2006. 